All Correspondence this week responds to Barbara Sahakian and Sharon MoreinZamir's Commentary 'Professor's little helper' (Nature 450, 1157-1159; 2007) and the related discussion at http://network.nature. com/forums/naturenewsandopinion.

This week, Nature launches an anonymous online survey to build on the informal questionnaire that the Commentary authors sent academics on the usage of brain-boosting drugs. In aggregate, the survey results will guide future editorial content on this topic. To take part, please visit: http://tinyurl.com/yq7nn3.

\section{The action of enhancers can lead to addiction}

SIR - Sahakian and Morein-Zamir revive questions about the widespread availability and diversion of prescription medications for non-clinical use in healthy individuals (Nature 450, 1157-1159; 2007). Such questions drove legislators to impose controls a few decades ago, when amphetamines and barbiturates were widely available.

Because the diversion of drugs is linked to their availability, the World Health Organization monitors their production and consumption by individual nations. In the United States, production of stimulant drugs has soared during the past two decades, and enough are now produced each year for the daily treatment of at least four million individuals. Even though stimulants and other cognitive enhancers are intended for legitimate clinical use, history predicts that greater availability will lead to an increase in diversion, misuse and abuse. Among high-school students, abuse of prescription medications is second only to cannabis use.

Although access to medications that improve our cognitive performance might be desirable in theory, these may have adverse medical consequences. Some limitations are necesssary, for these medications can be addictive. This is because cognitive enhancers such as the stimulants methylphenidate (Ritalin) and amphetamine amplify the activity of dopamine, a neurotransmitter that increases saliency, making cognitive tasks and everyday activities seem more interesting and rewarding. This learned experience can lead to abuse of the drug and to compulsive use and addiction in vulnerable people.

As we increase our knowledge of how the brain works, we may one day have safe interventions to improve cognition. In the meantime, we need to learn from history and avoid using them unnecessarily.

Nora D. Volkow ${ }^{\star}$, James M. Swanson'†

*National Institute on Drug Abuse, 6001 Executive Boulevard, Room 5274,

Bethesda, Maryland 20892, USA

†University of California at Irvine, Child Development Center, Irvine, California 92612, USA

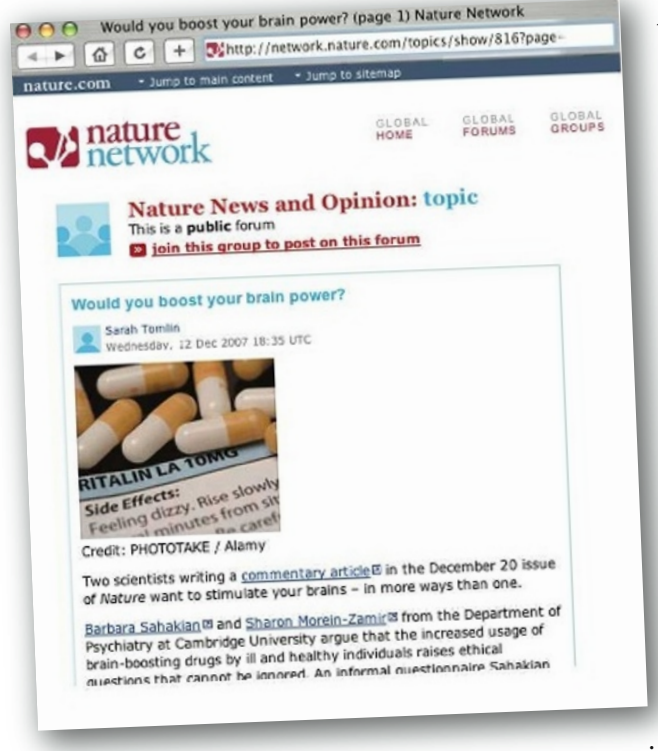

\section{Drugs can be used to treat more than disease}

SIR - Your fine Commentary draws attention to some important questions (Nature 450, 1157-1159; 2007). I agree with the point made by several commentators, that there is a need for better understanding of the long-term effects of using potential cognitive enhancers in an ecological setting. It is one thing to show a short-term positive effect on some artificial lab task; it is quite another to show that long-term use actually leads to sustainable performance gains on important real-world tasks, such as academic output. The former is easier to demonstrate, but the latter is what ultimately matters.

Unfortunately, progress on developing effective cognitive enhancers, and on understanding their long-term effects, is hampered by a shortage of focused research in this area. In general, the potential of enhancement medicine has yet to be fully appreciated.

Prevailing patterns of medical funding and regulation are organized around the concept of disease. Every pharmaceutical on the market with alleged cognitive-enhancing effects was developed as a treatment for some pathology. Its good effects on healthy adults' brains were discovered as fortuitous side effects. This disease-centred framework impedes the development of safe and effective enhancing medicines and has several consequences.

First, it makes funding hard to come by; it also makes it difficult to obtain regulatory approval for enhancement drugs. The result is that those who wish to research cognitive enhancement must often mask their work under the guise of addressing some 'respectable' disease.

Second, in order to gain access to the benefits of a cognitive enhancer, the user must first be classified as sick. This leads to the expansion of diagnostic categories and the invention of new pathological conditions - sometimes to cover cases that in earlier times would have been regarded as within normal human variation.

Third, it contributes to inequity in access. The main obstacle for someone who might be interested in trying modafinil or a related drug is not cost (which is similar to that of a large cup of coffee) but information: knowing that the drug exists and how to obtain it. This discriminates against people with little access to information.

With the cockcrow of enhancement medicine, we need to retool our regulatory paradigm. It is not only special occupations such as military commandos and air-traffic controllers that would benefit from good enhancement drugs.

Other jobs are just as important and intellectually taxing - including the jobs of many scientists and academics. Anything that can help our brains deal better with the complex challenges of the twenty-first century is to be not only welcomed but actively sought. But it will require substantial investment to develop interventions that are both safe and effective in long-term use.

\section{Nick Bostrom}

Future of Humanity Institute, James Martin 21st Century School, University of Oxford, Littlegate House, 16/17 St Ebbe's Street, Oxford OX11PT, UK

\section{Low dose of alertness drug counters 'family fatigue'}

SIR - I have been taking $50 \mathrm{mg}$ of modafinil almost daily for over a year. For most of my 74 years, I have struggled with fatigue and markedly reduced brain function every afternoon. This is part of my family history: my father and grandfather both structured afternoon naps into their schedules. I have found that caffeine and nicotine are either ineffective or cause a jittery nerviness. The side effects of the antidepressants I tried desipramine, Paxil and Wellbutrin - were not worth the minimal benefits.

At first, I used modafinil only when I desired an extended high level of attention. Previously, I could work competently on the fracture-mechanics of high-silica stone (while replicating ancient tool-flaking techniques) for about an hour. With modafinil, I could continue for almost three hours. It did not make me 'smarter', but extended the length of concentrated focus.

When I used it on a three-day crosscountry drive, I was not only more alert but found the journey more enjoyable and less tiring than previously.

I have not seen any data suggesting that modafinil is either habit-forming or easily abused (I have not looked for studies in 